\title{
Optical and Near-infrared High-resolution Spectroscopic Observations of Nova V2659 Cyg: Structure of Nova Ejecta and Origin of Two-distinct Velocity Systems
}

\footnotetext{
Akira Arai ${ }^{* a}$, Hideyo Kawakita ${ }^{a, b}$, Ashish Raj ${ }^{c}$, G. C. Anupama ${ }^{c}$, B. C. Lee ${ }^{d}$, Sohei Kondo $^{a}$, Yuji Ikeda $^{a, e}$, Naoto Kobayashi $^{a, f, g}$, Satoshi Hamano $^{a}$, Hiroaki Sameshima ${ }^{a}$, Kei Fukue $^{a}$, Noriyuki Matsunaga ${ }^{a, h}$, Chikako Yasui ${ }^{a, i}$, Natsuko Izumi ${ }^{a, i}$, Misaki Mizumoto $^{j}$, Shogo Otsubo ${ }^{a, b}$, Keiichi Takenaka ${ }^{a, b}$, Ayaka Watase ${ }^{a, b}$, Takafumi Kawanishi $^{a, b}$, Kenshi Nakanishi ${ }^{b}$, and Tetsuya Nakaoka ${ }^{b}$

${ }^{a}$ Laboratory of Infrared High-resolution spectroscopy (LiH), Koyama Astronomical Observatory, Kyoto Sangyo University, Motoyama, Kamigamo, Kita-ku, Kyoto 603-8555, Japan

${ }^{b}$ Department of Astrophysics and Atmospheric Sciences, Faculty of Sciences, Kyoto Sangyo University, Motoyama, Kamigamo, Kita-ku, Kyoto 603-8555, Japan

${ }^{c}$ Indian Institute of Astrophysics, II Block Koramangala, Bangalore 560034, India

${ }^{d}$ Korea Astronomy and Space Science Institute 776, Daedeokdae-ro, Yuseong-gu, Daejeon, Republic of Korea (34055)

e Photocoding, 460-102 Iwakura-Nakamachi, Sakyo-ku, Kyoto,606-0025, Japan

${ }^{f}$ Institute of Astronomy, School of Science, University of Tokyo,2-21-1 Osawa, Mitaka, Tokyo 181-0015, Japan

${ }^{g}$ Kiso Observatory, Institute of Astronomy, School of Science, The University of Tokyo, 10762-30 Mitake, Kiso-machi, Kiso-gun,Nagano, 397-0101, Japan

${ }^{h}$ Department of Astronomy, Graduate School of Science, University of Tokyo, Bunkyo-ku, Tokyo 113-0033, Japan

${ }^{i}$ National Astronomical Observatory of Japan, 2-21-1 Osawa, Mitaka, Tokyo 181-8588

${ }^{j}$ Centre for Extragalactic Astronomy, Department of Physics, University of Durham, South Road, Durham DH1 3LE, UK

E-mail: araibadcc.kyoto-su.ac.jp
} 
Two distinct absorption-line systems distinguished by radial velocities have often been observed in the optical high-resolution spectra of classical novae during their early decline phase. The origin of these absorption-line systems is under debate. We present optical high-resolution spectroscopic observations spectra of nova V2659 Cyg and discuss the temporal evolution of those absorption-line systems observed in this nova during its early decline phase. The observed temporal evolution of absorption-line profiles with relatively higher velocities (the high-velocity component) can be explained qualitatively by the clumpy ejecta and motion of the ionization fronts in the ejecta with time. Conversely, the low-velocity component may originate in the cool region compressed by the shock caused by collision between the fast nova wind and the slow expanding, equatorially focused dense ejecta. We also present high-resolution spectra of V2659 Cyg during its nebular phase in optical and near-infrared wavelength regions. Emission lines detected during the nebular phase also showed two velocity components, suggesting that the velocity structure of the ejecta during the nebular phase is similar to that during the early decline phase. The doublehorned profiles of emission lines with low velocities imply a ring-like distribution of materials with lower velocities. The observations during both the early-decline phase and the nebular phase support the multiple ejection of ejecta at a nova explosion, with different velocities.

The Golden Age of Cataclysmic Variables and Related Objects IV

11-16 September, 2017

Palermo, Italy

${ }^{*}$ Speaker. 


\section{Introduction}

Classical novae often display multiple absorption-line systems with different velocity components, caused by neutral atoms/ions existed in the nova ejecta along the line of sight (e.g., [四, 口, [ ] , 团] and references theirin), during their early-decline phase. Both spatial structure and velocity structure of the nova ejecta (especially, along the line-of-sight) could be investigated based on the absorption-line profiles of the systems. On the other hand, the emission-line profiles observed in novae during nebular phase (i.e., the nova ejecta are optically thin) also provide crucial information about the velocity structure of entire nova ejecta.

To date, several authors have proposed various physical models of nova ejecta based on obser-

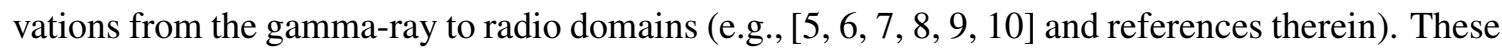
models can be classified into two groups: one assumes a single eruption for each nova outburst and other assumes multiple eruptions for each nova outburst. Recently, essentially important observations were reported for novae, that is, the detection of strong gamma-ray emissions (e.g., [Q], and references therein). The gamma-ray emissions in several novae strongly support the existence of shocks in nova ejecta.

Mason et al. in 2018 [ए]] and Shore et al. in 2011 [प] concluded that a single eruption at the nova outburst could explain the observed behavior if the temporal evolution of the recombination fronts within the nova ejecta was considered. In their scenario, materials can be ejected as multiple clumps and the nova ejecta are ballistic at the outburst. Mason et al. in 2018 [ए]] proposed persistent structures of nova ejecta that do not move in velocity space, i.e., in a Hubble/radial flow (or in a ballistic expansion). The density distribution of ejecta is stationary in the velocity space; their model is not compatible with the situation based on the nova wind. Even though the absorption features display apparent inward motion (or sometimes oscillations), they considered that such motion could be explained by a change in the ionization structure of the nova ejecta, i.e., moving recombination/ionization fronts in the ejecta. Their model does not require multiple ejections of shells or structures having increasing velocities. Furthermore, they claimed that collisions between clumps during the ejection and the initial expansion could explain the stochastic range of clump velocities as well as the gamma-ray emissions from novae. Shore et al. explained the change in the absorption-line systems found in the recurrent nova T Pyx [W], while Mason et al. discussed those in about V1369 Cen, T Pyx, V339 Del, and V959 Mon [ए]].

In contrast with the above scenario, several ejecta models have been proposed and they require multiple ejections of materials, such as the shells in the model proposed by McLaughlin [미, [13]. The most recent ejecta model explaining gamma-ray emissions from novae was proposed by $\mathrm{Li}$ et al. in 2017 ([0] and references therein). In their model, an isotropic nova wind with a faster expansion velocity collides with slower-velocity equatorially focused materials ejected before the initiation of the nova wind. Li et al. in 2017 [ [Q] considered a dense and equatorially focused slow outflow (possibly due to spiral-like mass loss) through the outer L2 Lagrange point of the binary system during the pre-maximum halt phase of the nova outburst or prior to the outburst. It is likely that such mass loss is important for slow novae, in which the nova photosphere expands slowly before it reaches the visual brightness maximum (it spends a longer time in the pre-maximum phase). The shock produced by the collision between these ejecta (the equatorially focused ejecta with slow velocities and the nova wind with faster velocities) can explain the gamma-ray emissions 
observed in novae ([Q, [4] and references therein). Their model also explains the formation of dust grains in novae during their early decline phase [15]. A dense region compressed by the shock could become cool enough to form dust grains in the later phase. That is, the ionization degree of the materials in this region is low during the early decline phase prior to the dust formation. In contrast with the equatorially focused ejecta, the materials blown with the nova wind may be diffuse and in lower densities unless they collide with the equatorially expanding ejecta. A wide range of ionization degrees could be achieved at different distances from the central nova in such a diffuse region [0]]. The clumpiness of the nova ejecta could be employed to explain the fine structure of the emission/absorption-profiles in velocity space.

In this article we report the temporal evolution of the absorption-line systems observed in V2659 Cyg after its outburst in 2014. This nova was discovered by Nishiyama and Kabashima [ए7] on UT Mar 31.79, 2014. Initial low-resolution spectroscopic studies [ए], ㅁ]] revealed that it is an Fe II-type nova according to the classification by Williams [ㅁ] ]. This nova displayed P-Cygni profiles for low-ionized species such as H I, Fe II, and O I with radial velocities $-480--650 \mathrm{~km} \mathrm{~s}^{-1}$. The slow decline rate of the optical light curve of this nova (Figure $\mathbb{Z}$ ), in addition to the slow expansion velocities, indicates that V2659 Cyg is a slowly evolving nova. The optical light curve is complicated and is classified as a "jitter" type [20]. Arai et al. in 2016 [可] have already reported a detailed analysis of the absorption lines found in the high-resolution spectra taken on May 13, 2014 (33 days after the visual brightness maximum). The authors argued for two distinct absorption-line systems in velocity space: a "low-velocity component (LVC)" and a "high-velocity component (HVC)". Here, we also report the high-resolution spectra taken on other dates, not only during the early decline phase but also during the nebular phase. We discuss the structure of the nova ejecta based on the observational features of the absorption-line systems in this nova from the viewpoints of two different models (single and multiple ejection scenarios).

\section{Observations}

High-resolution spectroscopic observations of the nova V2659 Cyg were conducted using several instruments, as summarized in Table 1. During the early decline phase, we used the HDS [R]] mounted on the 8-m Subaru telescope and the BOES [22] mounted on the 1.8-m telescope at BOAO in Korea, to obtain high-resolution spectra in the optical wavelength region. During the nebular phase of the nova, we also used the WINERED [23] mounted on a 1.3-m Araki telescope in Kyoto, Japan, to perform high-resolution near-infrared spectroscopic observations in addition to the optical observations by the BOES. We used the astronomical software package $\operatorname{IRAF}^{1}$ to reduce the spectroscopic data obtained by the BOES, based on the standard procedures for high-resolution echelle spectra. In the case of WINERED data, we used the customized pipeline software that has a capability to remove telluric absorption lines. The detail of the reduction process is described elsewhere (e.g., [24, [25]).

\footnotetext{
${ }^{1}$ IRAF is distributed by the National Optical Astronomy Observatory, which is operated by the Association of Universities for Research in Astronomy (AURA) under a cooperative agreement with the National Science Foundation.
} 


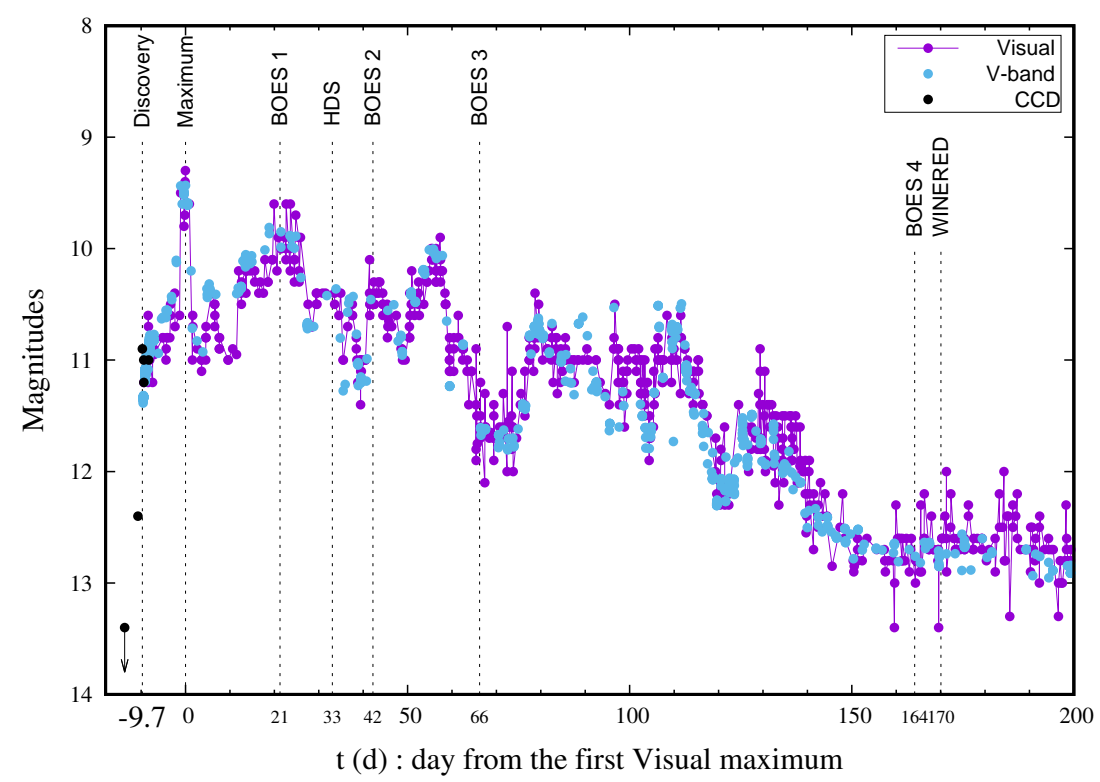

Figure 1: Optical light curves of V2659 Cyg. Labels with dotted line denote the dates of the discovery, the maximum, and our observations The labels "BOES1" - "BOES4" show the dates of our time-series observations with the BOES.

Table 1: Observational conditions.

\begin{tabular}{ccccl}
\hline $\begin{array}{c}\text { Date } \\
(\mathrm{UT})\end{array}$ & $\begin{array}{c}\mathrm{t} \\
(\text { days })^{a}\end{array}$ & $\begin{array}{c}\text { Total integration } \\
\text { time (s) }\end{array}$ & $\begin{array}{c}\text { Spectral resolving power } \\
(R=\lambda / \Delta \lambda)\end{array}$ & Instrument \\
\hline May 1, 2014 & 21 & 1,200 & 27,000 & BOES \\
May 13, 2014 & 33 & 600 & 72,000 & HDS \\
May 22, 2014 & 42 & 1,200 & 27,000 & BOES \\
Jun 15, 2014 & 66 & 1,200 & 27,000 & BOES \\
Sep 21, 2014 & 164 & 1,200 & 27,000 & BOES \\
Sep 27, 2014 & 170 & 4,400 & 20,000 & WINERED \\
\hline
\end{tabular}

${ }^{a} t=0$ for the visual brightness maximum.

\section{Results and Discussion}

\subsection{Line identification}

Arai et al. in 2016 [ [ $\mathbf{0}$ ] reported line identifications for the spectrum of V2659 Cyg, which was taken on May 22, $2014(t=33 \mathrm{~d})$. Here, in addition to this previous report, we report the line identifications of the nova for the high-resolution spectra taken by the BOES at other epochs (i.e., $t=21 \mathrm{~d}, 42 \mathrm{~d}$, and $66 \mathrm{~d}$ ) during the early decline phase in Figure $\mathrm{B}$. of the nova during the nebula phase, taken by both the BOES and the WINERED, are also shown with line identifications in Figure B.D. 


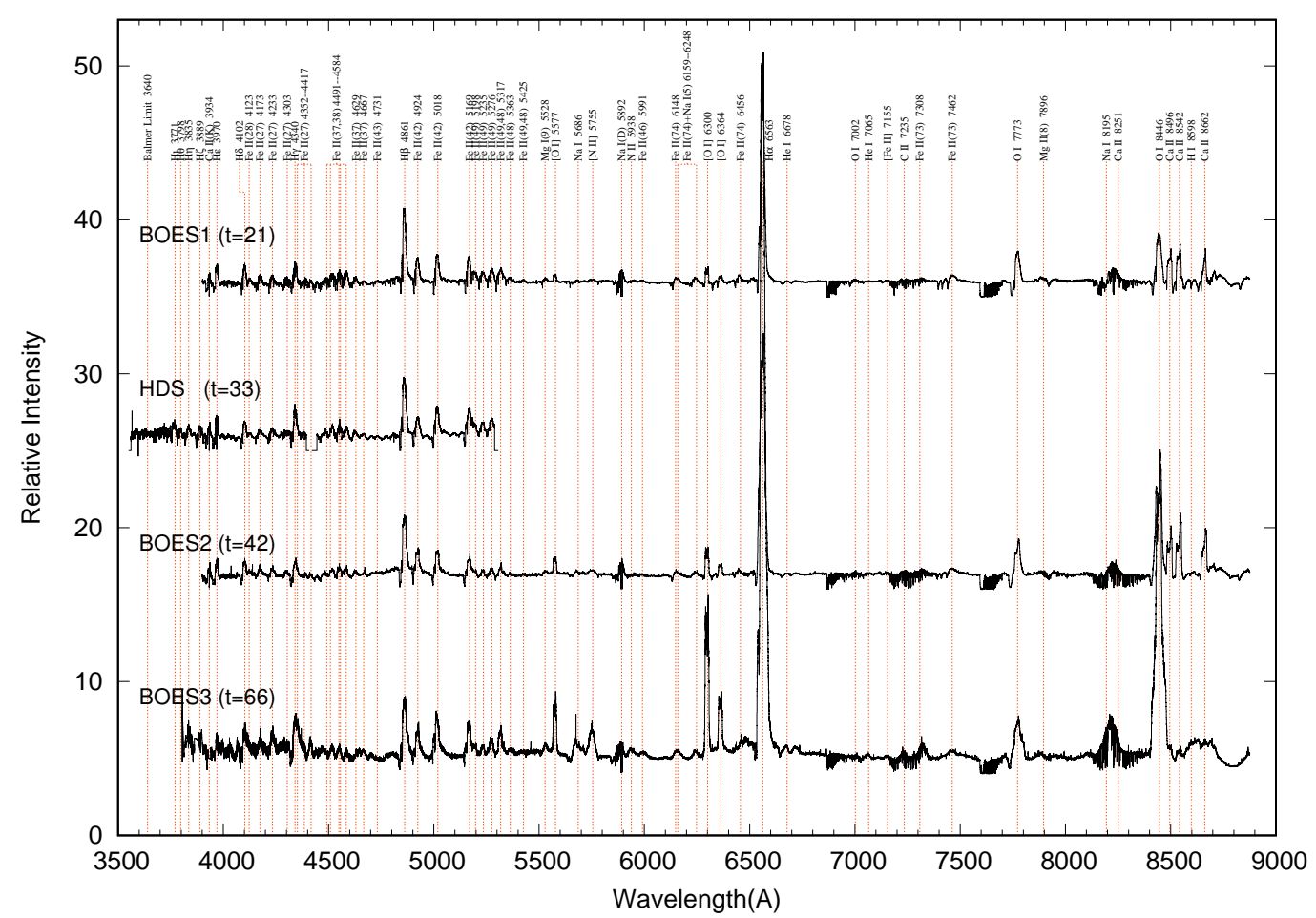

Figure 2: Optical spectra of the early decline phase of V2659 Cyg at $t=21 \mathrm{~d}, 33 \mathrm{~d}, 42 \mathrm{~d}$, and $66 \mathrm{~d}$. The dashed vertical lines indicate the positions of the identified emission lines.

\subsection{LVCs and HVCs during the Early Decline Phase}

Using the multi-epoch observations taken during the early decline phase of V2659 Cyg, the temporal evolution of the absorption-line systems of the LVCs and HVCs could be revealed. Fig-

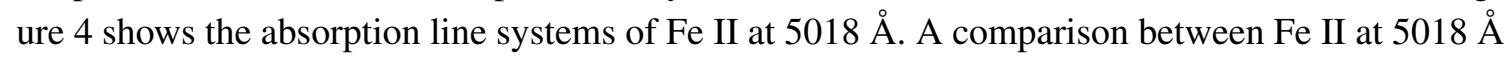
and Na I at $5889 \AA$ are also shown in Figure 田. The LVC (from $-600 \mathrm{~km} \mathrm{~s}^{-1}$ to $-800 \mathrm{~km} \mathrm{~s}^{-1}$ ) and HVC (from $-1000 \mathrm{~km} \mathrm{~s}^{-1}$ to $-1600 \mathrm{~km} \mathrm{~s}^{-1}$ ) are clearly recognized at all dates. The absorption-line profiles of both velocity systems changed during the early decline phase.

For the LVC, the absorption-line was relatively sharp earlier (FWHM $\sim 60 \mathrm{kms}^{-1}$ at $t=21$ d), became wider (FWHM $\sim 100 \mathrm{kms}^{-1}$ at $t=33 \mathrm{~d}$ ), and then took on a double-peaked shape later (at $t=42 \mathrm{~d}$ and $66 \mathrm{~d}$ ), as demonstrated in Figure $\mathrm{B}$ for Fe II at $5018 \AA$ A. Even for the large difference in the excitation potential of the lower energy levels for Fe II at $5018 \AA(10.3 \mathrm{eV})$ and for $\mathrm{Na} I$ at $5889 \AA(0.0 \mathrm{eV})$, the absorption-line profiles are very similar on the same dates, as shown in Figure $\mathrm{H}$. This indicates that the absorption lines formed via optically thick gas and that the absorption was saturated. Further, the bottom of the absorption did not reach the zero level, i.e., the covering factor of the gas was not unity. It is likely that dense blobs of nova ejecta absorbed 

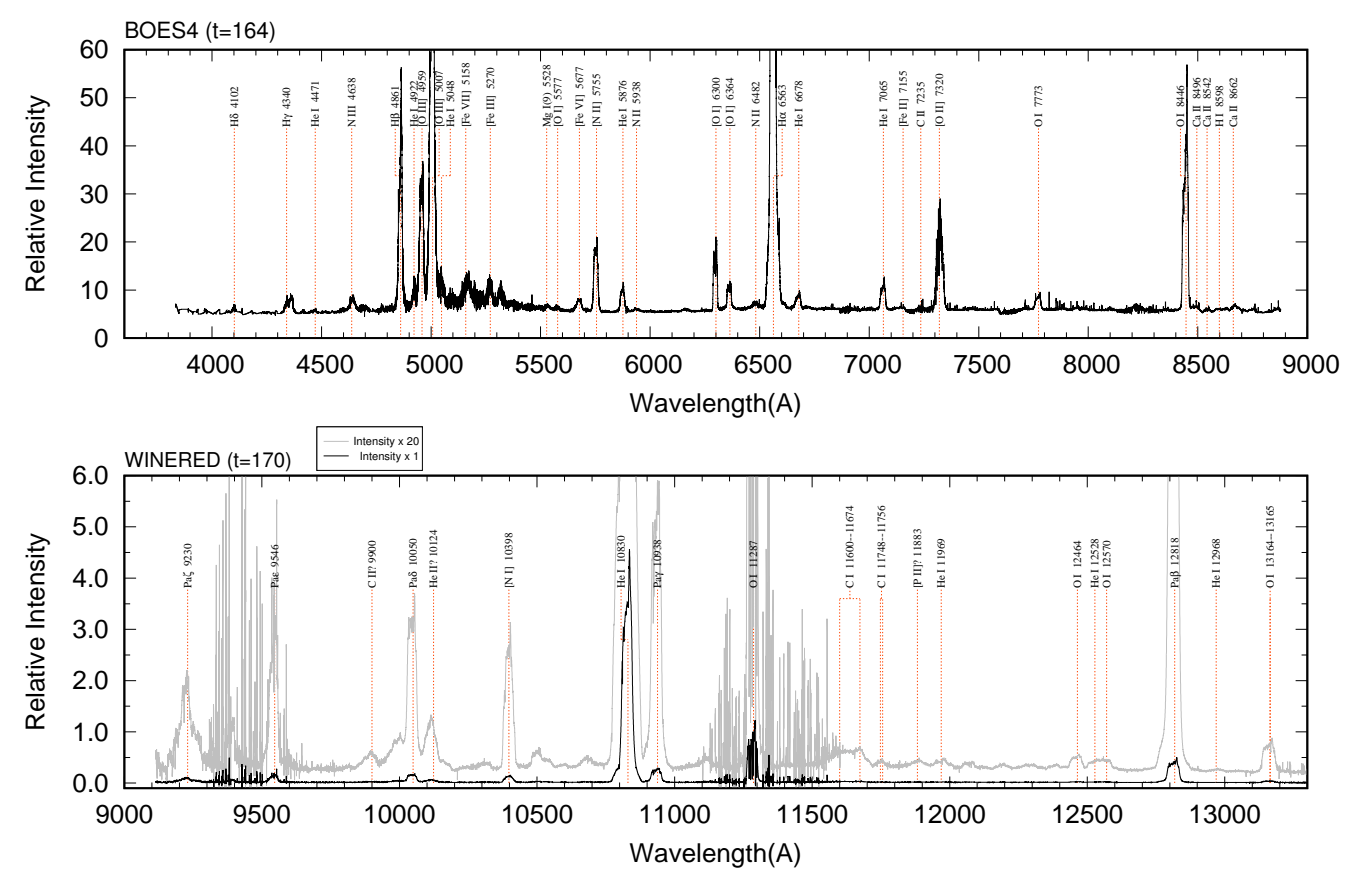

Figure 3: Optical and near infrared spectra of the nebular phase of V2659 Cyg at $t=164 \mathrm{~d}$ and $170 \mathrm{~d}$. The dashed vertical lines indicate the positions of the identified emission lines. The grey spectrum in the bottom panel shows the relative flux of nearinfrared data scaled by 20 times. Line idenifications of the near-infrared spectrum was refered to [26, 27, 28, 29].

light from the nova photosphere. Arai et al. in 2016 [团] proposed the same picture because the absorption-line profiles of Fe II with different $g f$-values displayed similar profiles at $t=33 \mathrm{~d}$ (see Figure 4 in [四]). The acceleration of the absorption profile was recognized as observed in other novae [U], as shown in Figure [1]. Note that the fastest velocity of the LVC appears to terminate as approximately $-900 \mathrm{~km} \mathrm{~s}^{-1}$ at $t=66 \mathrm{~d}$, in contrast to the monotonic increase in the slowest velocity of the LVC during the period of our observations.

Regarding the origin of LVC in V2659 Cyg, absorption lines of LVC originated from energy levels with low excitation potential $(\leq 4 \mathrm{eV})$ of lowly-ionized Fe-peak and s-process elements as also reported in other novae, e.g., Sc II, Ti II, V II, Cr II, Fe II, Sr II, Y II, Zr II, Ba II, and Mn II

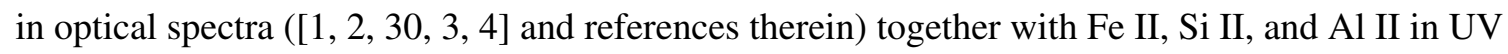
spectra [3]. Strong emission lines in novae such as H I, He I, Na I, Mg I, Mg II, Ca II, O I, also show the absorption system with similar velocities in the novae including V2659 Cyg.

The "low-ionization" is probably a key to understand the origin of LVC. The multiple ejection scenario proposed by $\mathrm{Li}$ et al. in 2017 [Q⿴囗⿰丿㇄口] assumed a dense and equatorially focused slow outflow and an isotropic nova wind with faster velocities (leading to the collision between the slow outflow and the nova wind). The equatorially focused region is compressed and becomes cool due to the shock at the collision, sometimes followed by the dust formation during early decline phase [[15]. 

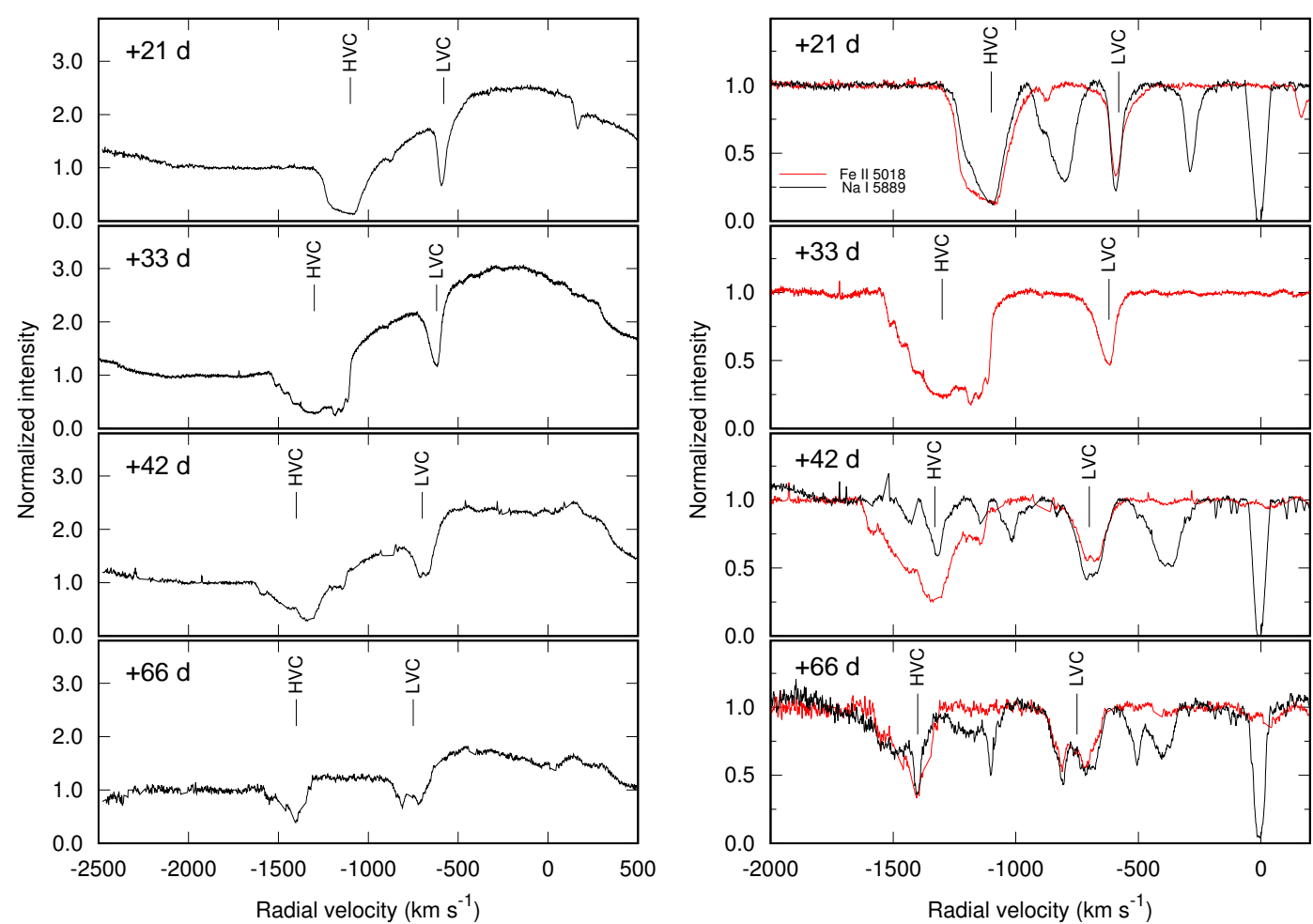

Figure 4: The left panel shows absorption systems (LVCs and HVCs) of Fe II $5018 \AA$. The right panel shows a comparison of LVCs and HVCs between Fe II $5018 \AA$ and Na I $5889 \AA$. In the right panel, spectra are normalized by the polynomical functions fit with the emission profile.

Thus, the feature of "low-ionization" is consistent with the hypothesis that the LVC originates from such equatorially focused outflow compressed by the collision between the slow outflow and the fast nova wind.

In contrast to the LVC, the HVC displayed very different, complicated line profiles. The existence of different velocity components (a multi-peak profile) is indicative of clumpy structure of the nova ejecta. Figure $[$ shows the temporal change in the absorption-line profile in the LVC and the HVC. An apparent acceleration of the absorption-line profiles was recognized for the LVC; however, a much more complicated situation exists for the HVC. Note that the step-like features for the range from $-1400 \mathrm{~km} \mathrm{~s}^{-1}$ to $-1600 \mathrm{~km} \mathrm{~s}^{-1}$ (likely composed of multiple absorption lines) shows similar velocities at each peak between $t=42 \mathrm{~d}$ and $66 \mathrm{~d}$. Stationary velocity structure of the nova ejecta is supported by this observational fact. As discussed by Mason in 2018 [ए]] and Shore et al. in 2011 [U]], the apparent change of the absorption-line system may be explained by the moving recombination/ionization fronts in the nova envelope.

The slowest velocity of HVC absorption increased monotonically although the fastest velocity terminated in later epochs as for the LVC (Figure Bla). The fastest velocity in the latest epoch $\left(\sim-1600 \mathrm{~km} \mathrm{~s}^{-1}\right)$ may indicate the maximum velocity of the ejecta. Regarding the origin of HVC in the nova, HVC has a wide range in ionization in novae (e.g., C IV, N V, Al III, C II and O I 

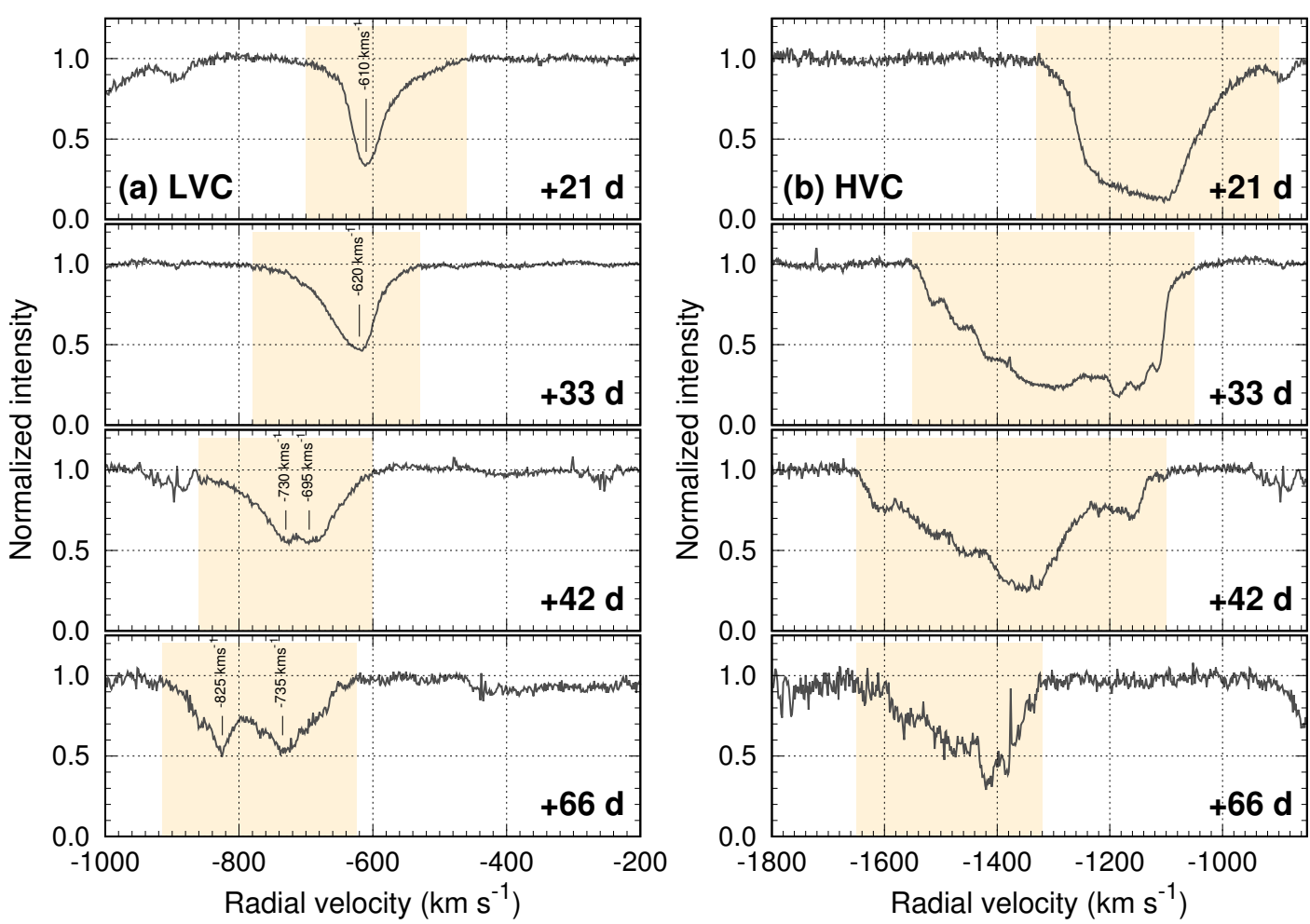

Figure 5: Temporal developments of (a) the LVC and (b) the HVC at Fe II $5018 \AA$ from $t=21 \mathrm{~d}$ to $66 \mathrm{~d}$. Shaded region (in orange) denote the approximate widths of the velocity range for each absorption feature.

absorption lines were recognized in UV spectra [B]]) as pointed out by Williams \& Mason (2010). A wide range of ionization degrees could be achieved in nova envelope (without compression by the shock) at different distances from the central nova [ए6].

\subsection{Emission Profiles of the Nebular Phase Spectra}

Basically the nebular phase is observationally defined as the period when the [O III] emission lines are stronger than the $\mathrm{H} \beta$ emission, indicating the nova ejecta is optically thin. In the case of V2659 Cyg, at $t=164 \mathrm{~d}$, the nebular lines such as [O III] lines of the nova had already been stronger than the $\mathrm{H} \beta$. Here we present the high-resolution spectra of the nova on $t=164$ and 170 d.

The spatial distribution of the nova ejecta of V2659 Cyg during the nebular phase can be inferred from the emission-line profiles. Figure $\mathbf{G}$ and $\square$ show the emission-line spectra of [O I] at $6300 \AA$ [O III] at $5007 \AA(t=164 \mathrm{~d})$, and He I at $10830 \AA(t=170 \mathrm{~d})$. Note that the telluric absorption lines were not corrected in Figure 6 (for [O I] and [O III]), but almost corrected in Figure $\square$ (for He I). The peaks at $-450 \mathrm{kms}^{-1}$ and $+170 \mathrm{kms}^{-1}$ are clear in these emission-line profiles. Here we concentrate on the emission-line profile of He I because the telluric absorption lines are well corrected and the continuum fitting is easier due to less contamination by other emission lines for the He I emission. The observed emission-line profile of He I is double-horned 
and it can be decomposed by two velocity components (with FWHMs of $\sim 700$ and $\sim 1700 \mathrm{kms}^{-1}$ ) along with a double-peaked profile as shown in Figure $\square$. The expansion velocities estimated from these FWHMs are in good agreement with the terminal velocities of the fastest edge in LVCs $\left(\sim-900 \mathrm{~km} \mathrm{~s}^{-1}\right)$ and in HVCs $\left(\sim-1600 \mathrm{~km} \mathrm{~s}^{-1}\right)$ at $t=66 \mathrm{~d}$, respectively (as mentioned in the previous section, see Figure (1). These results indicate the existence of two distinct velocity systems for the nova ejecta.

Mason et al. in 2018 [ए]] calculated the emission-line profiles of the nova during the nebular phase for ejecta distributions with various opening angles as observed from various lines-of-sight with respect to the orbital plane of the central binary system of the nova. Based on a comparison with their results, the ejecta distribution of V2659 Cyg might have a nonspherical biconical structure. Further, the emission-line profiles of [O I], [O III] and He I display complicated velocity structures (especially multiple velocity peaks for the narrower component) and suggest of a clumpy, multiple ring structure of the nova ejecta.

As mentioned in Section 3.2, a complicated multi-peak structure for the HVC was seen during the early decline phase. However, these systems were not seen during the nebular phase. This difference is probably explained by the difference in the contributing regions of the ejecta for absorption and emission. The absorption seen during the early decline phase was caused by the gas along the line-of-sight only (almost moving toward the observer) against the background light source. On the other hand, the emission seen during the nebular phase was produced from the gas of entire ejecta (not limited for the line-of-sight). Therefore, only smaller number of clumps contributed to the absorption during the early decline phase (these clumps could be distinguished in the velocity space, as multiple absorption peaks) while all clumps contributed to the emission during the nebular phase (those clumps could not be separated). This hypothesis should be reexamined for future observations of different novae.

In summary, our observational facts strongly indicate that two distinct velocity components (the LVC and the HVC) of the nova envelope of V2659 Cyg existed until its nebular phase, at least. The origin of those ejecta might be a multiple eruption of materials from the nova, with different velocities.

\section{Conclusions}

We report the high-resolution spectra of the nova V2659 Cyg from the early decline phase to the nebular phase and discuss the velocity structure of the nova envelope. During its early decline phase, the nova displayed absorption-line profiles corresponding to two-distinct velocity components (the LVC and the HVC) along with sub-peaks indicative of a clumpy nova envelope structure. The velocities for the absorption components changed during our observations. In general, the apparent outward motion of the absorptions, i.e., their acceleration, was recognized. However, the fastest velocities of the LVC and HVC components appear to terminate at $\sim 900 \mathrm{~km} \mathrm{~s}^{-1}$ and $\sim 1600 \mathrm{~km} \mathrm{~s}^{-1}$ in the later phase. The emission-line profiles seen during the nebular phase of 


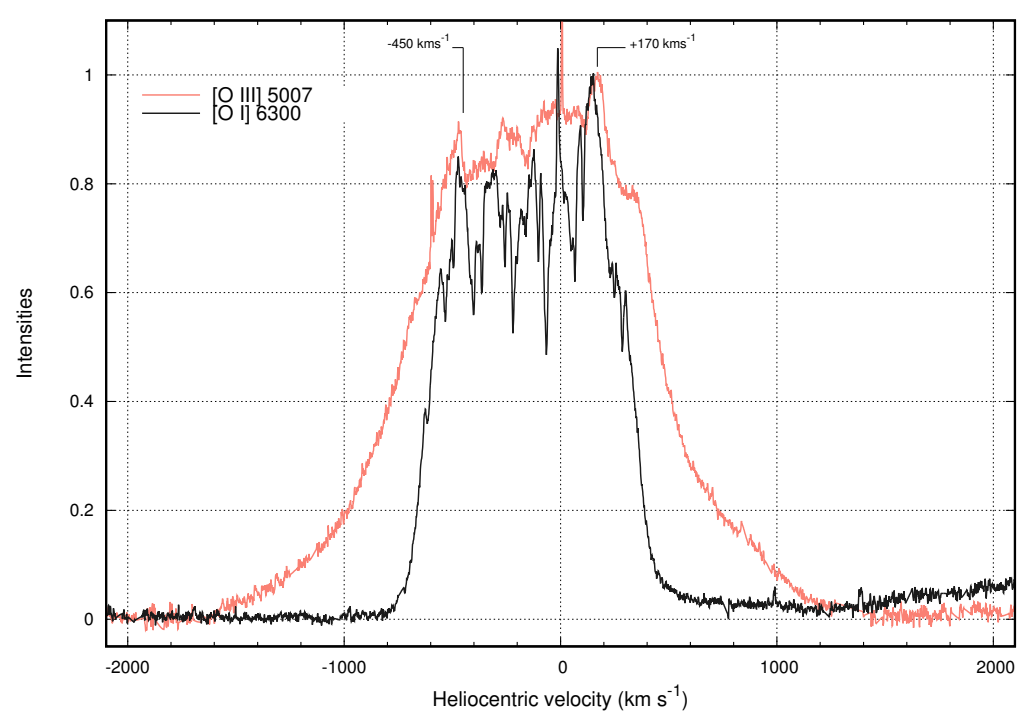

Figure 6: Forbidden emission lines, [O I] $6300 \AA$ and [O III] $5007 \AA$ on $t=164 \mathrm{~d}$ obtained by the BOES. Telliric absorption lines are not corrected for those profiles.

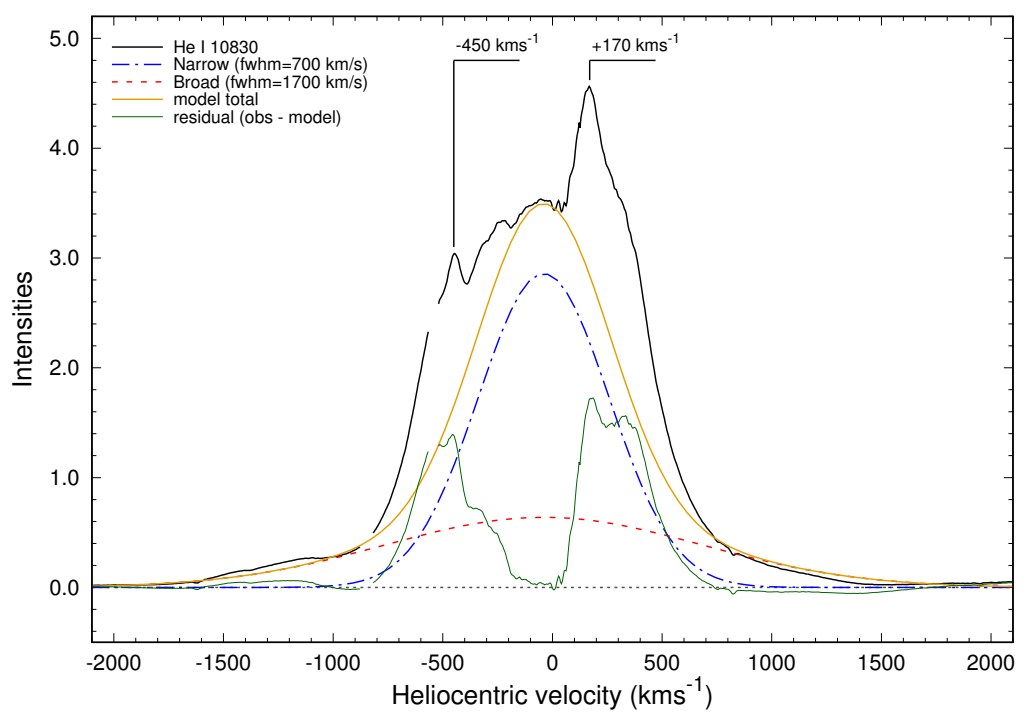

Figure 7: Line profile of He I $10830 \AA$ on $t=170 \mathrm{~d}$. The dashed blue and red lines are narrow ( $F W H M$ of $700 \mathrm{kms}^{-1}$ ) and broad ( $F W H M$ of $1700 \mathrm{kms}^{-1}$ ) components de-composed by Gaussian fittings, respectively. 
V2659 Cyg also indicate the existence of two velocity components with FWHMs of $\sim 700 \mathrm{~km} \mathrm{~s}^{-1}$ and $\sim 1700 \mathrm{~km} \mathrm{~s}^{-1}$, consistent with the terminal velocities found during the early-decline phase as described above. A clumpy structure is also implied for the envelope materials in the LVC system (but not obviously for the HVC system).

We conclude that the nova envelope of V2659 Cyg has two-distinct velocity components and is thought to be clumpy. The velocity structure might be stationary, and the apparent motion of the absorption lines during the early decline phase may be explained by the changing recombination/ionization fronts in the nova envelope as the envelope thinned out.

\section{Acknowledgements}

This study is financially supported by JSPS KAKENHI (16684001) Grant-in-Aid for Young Scientists (A), JSPS KAKENHI (20340042) Grant-in-Aid for Scientific Research (B), JSPS KAKENHI (26287028) Grant-in-Aid for Scientific Research (B), JSPS KAKENHI (21840052) Grantin-Aid for Young Scientists (Start-up), and MEXT Supported Program for the Strategic Research Foundation at Private Universities, 2008-2012 (No. S0801061) and 2014-2018 (No. S1411028). A.A. is supported by a grant received from the Sumitomo Foundation (No.141404). K.F. is supported by KAKENHI (16H07323) Grant-in-Aid for Research Activity start-up. S.H. is supported by Grant-in-Aid for JSPS Fellows Grant No. 13J10504. N.K. is supported by JSPS-DST under the Japan-India Scinece Cooperative Programs during 2013-2015 and 2016-2018.

\section{References}

[1] R. Williams, E. Mason, M. Della Valle and A. Ederoclite, Transient Heavy Element Absorption Systems in Novae: Episodic Mass Ejection from the Secondary Star, ApJ685(2008) 451] [0805.1372].

[2] A. Raj, N. M. Ashok, D. P. K. Banerjee, U. Munari, P. Valisa and S. Dallaporta, V496 Scuti: an Fe II nova with dust shell accompanied by CO emission, MNRAS 425 (2012) 2576 [1207.4876].

[3] A. Tajitsu, K. Sadakane, H. Naito, A. Arai, H. Kawakita and W. Aoki, The ${ }^{7}$ Be II Resonance Lines in Two Classical Novae V5668 Sgr and V2944 Oph, ApJ818(2016) एप].

[4] A. Arai, H. Kawakita, Y. Shinnaka and A. Tajitsu, Origins of Absorption Systems of Classical Nova V2659 Cyg (Nova Cyg 2014), ApJ 830 (2016) 30.

[5] D. B. McLaughlin, The spectrum of Nova Geminorum, 1912, Publications of Michigan Observatory 9 (1965) 113.

[6] C. D. Gill and T. J. O’Brien, Emission-line profiles from model nova shells, MNRAS 307 (1999) 677.

[7] G. H. Schaefer, T. T. Brummelaar, D. R. Gies, C. D. Farrington, B. Kloppenborg, O. Chesneau et al., The expanding fireball of Nova Delphini 2013, Nature 515 (2014) 234.

[8] B. D. Metzger, R. Hascoët, I. Vurm, A. M. Beloborodov, L. Chomiuk, J. L. Sokoloski et al., Shocks in nova outflows - I. Thermal emission, MNRAS 442 (2014) 713 [11403.1579].

[9] K.-L. Li, B. D. Metzger, L. Chomiuk, I. Vurm, J. Strader, T. Finzell et al., A nova outburst powered by shocks, Nature Astronomy 1 (2017) 697. 
[10] E. Mason, S. N. Shore, I. De Gennaro Aquino, L. Izzo, K. Page and G. J. Schwarz, V1369 Cen High-resolution Panchromatic Late Nebular Spectra in the Context of a Unified Picture for Nova Ejecta, ApJ 853(2018) 27.

[11] S. N. Shore, T. Augusteijn, A. Ederoclite and H. Uthas, The spectroscopic evolution of the recurrent nova T Pyxidis during its 2011 outburst. I. The optically thick phase and the o A\&A 533(2011)L8 [L108.3505].

[12] D. B. McLaughlin, A model of a nova outburst, Popular Astronomy 58 (1950) 50.

[13] D. B. Mclaughlin, Problems in the Spectra of Novae, Publications of the Astronomical Society of the Pacific 62 (1950) 185.

[14] T. Finzell, L. Chomiuk, B. D. Metzger, F. M. Walter, J. D. Linford, K. Mukai et al., A Detailed Observational Analysis of V1324 Sco, the Most Gamma-Ray- luminous Classical Nova to Date, ApJ 852 (2018) 108.

[15] A. M. Derdzinski, B. D. Metzger and D. Lazzati, Radiative shocks create environments for dust formation in classical novae, MNRAS 469 (2017) 핀.

[16] H. K. B. Beck, P. H. Hauschildt, H. P. Gail and E. Sedlmayr, Ionization and temperature of nova shells. II. The influence of realistic photospheric radiation fields., A\&A 294 (1995) 195.

[17] K. Nishiyama, F. Kabashima, G. Masi, F. Nocentini, P. Schmeer, W. Vollmann et al., Nova Cygni 2014 = Pnv J20214234+3103296, Central Bureau Electronic Telegrams 3842 (2014) 1.

[18] A. Raj, U. Munari, B.-C. Lee, S. C. Kim, S.-J. Kim and C.-K. Sim, High-resolution spectroscopy of Nova Cyg 2014, The Astronomer's Telegram 6181 (2014) 1.

[19] R. E. Williams, The Formation of Novae Spectra, AJT104 (1992) 725.

[20] R. J. Strope, B. E. Schaefer and A. A. Henden, Catalog of 93 Nova Light Curves: Classification and Properties, AJ 140 (2010) 34 [10004.3698]].

[21] K. Noguchi, W. Aoki, S. Kawanomoto, H. Ando, S. Honda, H. Izumiura et al., High Dispersion Spectrograph (HDS) for the Subaru Telescope, Publications of the Astronomical Society of Japan 54(2002) 855 .

[22] K.-M. Kim, I. Han, G. G. Valyavin, S. Plachinda, J. G. Jang, B.-H. Jang et al., The BOES Spectropolarimeter for Zeeman Measurements of Stellar Magnetic Fields, Bublications of the Astronomical Society of the Pacific 119 (2007) 1052 [0707.4220].

[23] Y. Ikeda, N. Kobayashi, S. Kondo, S. Otsubo, S. Hamano, H. Sameshima et al., High sensitivity, wide coverage, and high-resolution NIR non-cryogenic spectrograph, WINERED, in Ground-based and Airborne Instrumentation for Astronomy VI, vol. 9908, p. 99085Z, Aug., 2016, DOIl.

[24] S. Hamano, N. Kobayashi, S. Kondo, Y. Ikeda, K. Nakanishi, C. Yasui et al., Near-infrared Diffuse Interstellar Bands in 0.91-1.32 $\mu \mathrm{m}$, ApJ 800 (2015) 137 [1412.3578].

[25] S. Hamano, N. Kobayashi, S. Kondo, H. Sameshima, K. Nakanishi, Y. Ikeda et al., Near Infrared Diffuse Interstellar Bands Toward the Cygnus OB2 Association, ApJ 821 (2016) 42.

[26] D. Pequignot and J. P. Baluteau, The 680-1050 MM recombination spectrum of hydrogen and helium in the planetary nebula NGC 7027., A\&A 206 (1988) 298. 
[27] R. J. Rudy, P. Erwin, G. S. Rossano and R. C. Puetter, 0.8-1.6 Micron Spectroscopy of the Planetary Nebula NGC 7027, ApJ 384(1992) 536.

[28] D. P. K. Banerjee and N. M. Ashok, Near-infrared properties of classical novae: A perspective gained from Mount Abu Infrared Observatory, Bulletin of the Astronomical Society of India 40 (2012) 243 [ए306.0343].

[29] A. Evans, D. P. K. Banerjee, R. D. Gehrz, V. Joshi, N. M. Ashok, V. A. R. M. Ribeiro et al., Rise and fall of the dust shell of the classical nova V339 Delphini, MNRAS 466 (2017) 4221].

[30] L. Izzo, M. Della Valle, E. Mason, F. Matteucci, D. Romano, L. Pasquini et al., Early Optical Spectra of Nova V1369 Cen Show the Presence of Lithium, ApJ 808 (2015) L14.

[31] A. Cassatella, H. J. G. L. M. Lamers, C. Rossi, A. Altamore and R. González-Riestra, A study of the expanding envelope of Nova V1974 Cyg 1992 based on IUE high resolution spectroscopy, A\&A 420 (2004)57] [astro-ph/0403288]. 